\title{
IS-M 854 \\ CONF. $960334--3$ \\ Comparison of Different Pressing Techniques for the Preparation of n-type Silicon-Germanium Thermoelectric Alloys
}

\author{
by J. L. Harringa and B. A. Cook \\ Ames Laboratory, Iowa State University, Ames, IA 50010-3020, USA.
}

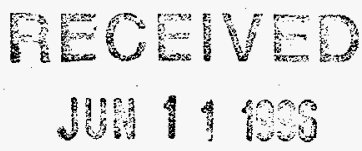

\begin{abstract}
Improvements to state-of-the-art $\mathrm{Si}_{80} \mathrm{Ge}_{20}$ thermoelectric alloys have been observed in laboratory-scale samples by the application of the powder metallurgical techniques of mechanical alloying and hot pressing. Incorporating these improvements in large scale compacts for the production of thermoelectric generator elements is the next step in achieving higher efficiency RTG's. This paper discusses consolidation of large quantities of mechanically alloyed powders into production size compacts. Differences in thermoelectric properties are noted between the compacts prepared by the standard technique of hot uniaxial pressing and hot isostatic pressing. Most significant is the difference in carrier concentration between the alloys prepared by the two consolidation techniques.
\end{abstract}

\section{Introduction}

Silicon-germanium thermoelectric alloys have been used for many years in radioisotope thermoelectric generators for space applications. These alloys are typically manufactured via a melting, grinding, and hot pressing sequence [1]. Due to the large separation between the solidus and liquidus in the Si-Ge phase system, dendritic segregation is prevalent in these alloys. This requires that multiple hot pressing and grinding sequences be carried out in order to homogenize the alloys. Cook et al. [2] have shown the viability of mechanical alloying (MA) using a high energy ball mill applied to heavily doped $\mathrm{Si}-\mathrm{Ge}$ alloys for thermoelectric applications. The advantage of MA over conventional techniques is that alloying is carried out at ambient temperatures and a homogeneous alloy is produced in a single MA + hot uniaxial pressing (HUP) step. A further advantage is that volatile phosphorous, the n-type dopant, is incorporated into the alloy at room temperature in a sealed vial rather than to a melt at high temperature as is conventionally done. Cook et al. [3] have shown that second phase oxygen has deleterious effects on the thermoelectric properties of n-type Si-Ge alloys. The MA process allows for the handling of precursor materials and the alloyed powders under a purified $\mathrm{He}$ atmosphere provided by a glove box, thus avoiding the reaction of the fine powders with air. Conventionally prepared material can contain a much higher level of second phase oxygen than MA materials due to powder handling.

Mechanical alloying has been applied to Si-Ge thermoelectric alloys on a laboratory scale. This is due to the fact that the milling equipment, a Spex $8000 \mathrm{mixer} / \mathrm{mill}$, is capable of alloying only 5-6 grams of powder at Samples produced at the Ames Laboratory consist of vacuum hot pressed $1.27 \mathrm{~cm}$ diameter cylinders approximately $2.5 \mathrm{~cm}$ in length. The scaleup from laboratory to industrial production requires larger volumes of powder to prepare large hot pressed billets called "pucks" from which thermoelements for RTG's are machined. Other milling equipment is available that is capable of producing $\mathrm{MA}$ powder in larger quantities. To avoid the problem of oxygen contamination, preparation of Si-Ge pucks needed to be carried out at the Ames Laboratory. Hot isostatic pressing (HIP) was thus attempted.

\section{Experimental}

As reported by Cook et al. [4] MA has been applied on the laboratory scale to prepare $\mathrm{GaP}$ and $\mathrm{P}$ doped $\mathrm{n}$-type SiGe alloys with high figures of merit. Production of the MA. powder for large scale HIPing was carried out in the same manner as for the laboratory scale alloys. Initial components consisted of Si (Hemlock Semiconductor), Ge (Cabot Berylco), GaP (Cerac) and P (Cerac). All of the starting materials were added to a round ended hardened steel vial as +20 mesh chunks in order to lower the oxygen content in the alloys introduced from surface oxide layers. The material was ball milled for 2 hours to form a mixed powder. Five grams of the premilled powder were removed and added to a flat ended hardened steel vial. This powder was then milled for 6 hours to form an alloy. Multiple MA millings were used to accumulate powder for HIPing whereas one 6 hour MA milling provided all the powder necessary for one HUP consolidation. Handling of the elemental components and MA powder was performed in a purified He filled glove box. Table 1 summarizes the sample compositions and consolidation technique. The greek letter designates an individual HUP sample consolidation.

\section{HUP Samples}

HUP samples were prepared by loading four grams of MA powder were loaded into a $1.27 \mathrm{~cm}$ graphoil lined graphite die. The powder was pressed in an $R F$ vacuum furnace at $1130^{\circ} \mathrm{C}$ and an applied pressure of $140 \mathrm{MPa}$ for 45 minutes after which the pressure was released and the sample was allowed to furnace cool. Samples were then sectioned from the compact and given a "reset" heat treatment at $1050^{\circ} \mathrm{C}$ for 20 minutes. One sample, $\mathrm{A} 92 \beta$, was pressed for 60 minutes. 


\section{HIP Samples}

HIP samples were prepared by packing the MA powder into a cylindrical graphoil lined Ta HIP vessel. A Ta lid was pressure fit into the vessel and e-beam welded under vacuum. Each HIP vessel was held in the vacuum port of the e-beam welder overnight to remove residual $\mathrm{He}$ in the vessel before the lid was e-beam welded in place.

Two types of HIP vessels were used. Initial HIP vessels were cylindrical $T a$ vessels with wall thickness of 0.05 $\mathrm{cm}$, diameter of $1.91 \mathrm{~cm}$, and height of $3.8 \mathrm{~cm}$. This vessel type was used on samples designated HIP-2, HIP-5a, and
$20 \mu \mathrm{m}$ range with most grains approximately $15 \mu \mathrm{m}$. The transverse section showed a very fine grain structure with grain sizes of $\sim 1 \mu \mathrm{m}$ and an occasional large grain of $\sim 20 \mu \mathrm{m}$.

The microstructure of HIP-10 exhibited longitudinal grains that appear to be in the $10-20 \mu \mathrm{m}$ range. The grains in the transverse section are larger and in the range of $\sim 12-\sim 40$ $\mu \mathrm{m}$. The difference in grain size between the transverse and longitudinal directions does not appear to be as significant as for the HIP-5a compact. It would thus appear that the aspect ratio of the HIP vessel greatly influences the size and shape of the grains. It is assumed that HIP-2 has the same type of microstructure as HIP-Sa due to the identical type HIP vessel

Table 1 Composition and consolidation conditions for $\mathrm{n}$-type $\mathrm{Si}_{80} \mathrm{Ge}_{20}$ alloys. All alloys were consolidated at $1130^{\circ} \mathrm{C}$ and $140 \mathrm{MPa}$.

\begin{tabular}{cccc}
\hline \hline Sample & Composition & Consolidation Method & $\begin{array}{c}\text { Consolidation Time } \\
\text { (minutes) }\end{array}$ \\
\hline $\mathrm{A} 92 \alpha$ & $\mathrm{Si}_{0.785} \mathrm{Ge}_{0.196 \mathrm{Ga}_{0.006} \mathrm{P}_{0.019}}$ & HUP & 45 \\
$\mathrm{~A} 92 \beta$ & $\mathrm{Si}_{0.785} \mathrm{Ge}_{0.196} \mathrm{Ga}_{0.006} \mathrm{P}_{0.019}$ & HUP & 60 \\
$\mathrm{~A} 93 \alpha$ & $\mathrm{Si}_{0.780} \mathrm{Ge}_{0.195} \mathrm{Ga}_{0.013} \mathrm{P}_{0.025}$ & HUP & 45 \\
$\mathrm{~A} 95 \alpha$ & $\mathrm{Si}_{0.778} \mathrm{Ge}_{0.195} \mathrm{Ga}_{0.013} \mathrm{P}_{0.028}$ & HUP & 45 \\
$\mathrm{~A} 96 \beta$ & $\mathrm{Si}_{0.776} \mathrm{Ge}_{0.194} \mathrm{Ga}_{0.013} \mathrm{P}_{0.030}$ & HUP & 45 \\
$\mathrm{~A} 99 \alpha$ & $\mathrm{Si}_{0.776} \mathrm{Ge}_{0.194} \mathrm{Ga}_{0.008} \mathrm{P}_{0.030}$ & HUP & 45 \\
$\mathrm{HIP}-2$ & $\mathrm{Si}_{0.785} \mathrm{Ge}_{0.196} \mathrm{Ga} 0.006 \mathrm{P}_{0.019}$ & HIP & 45 \\
HIP-5a, 5b & $\mathrm{Si}_{0.788} \mathrm{Ge}_{0.197} \mathrm{Ga}_{0.005} \mathrm{P}_{0.015}$ & HIP & 45 \\
HIP-7 through & $\mathrm{Si}_{0.788} \mathrm{Ge}_{0.197} \mathrm{Ga}_{0.005} \mathrm{P}_{0.015}$ & HIP & 45 \\
HIP-18 & & & 45 \\
A101-1 through & $\mathrm{Si}_{0.791} \mathrm{Ge}_{0.198} \mathrm{Ga} 0.004 \mathrm{P}_{0.011}$ & HIP & \\
A101-4 & & & \\
\hline \hline
\end{tabular}

HIP- 5 b. The final vessels used for the production of large compacts were constructed from $0.05 \mathrm{~cm}$ thick $\mathrm{Ta}$ with 5.08 $\mathrm{cm}$ diameter $\times 3.81 \mathrm{~cm}$ length. These vessels were filled with approximately $80 \mathrm{gm}$ of packed powder. The vessel was HIPed at $1130^{\circ} \mathrm{C}$ for 45 minutes under an applied pressure of $137 \mathrm{MPa}$. The furnace temperature was ramped from 25 $900^{\circ} \mathrm{C}$ at $10^{\circ} \mathrm{C} / \mathrm{min}, 900-1130^{\circ} \mathrm{C}$ at $5^{\circ} \mathrm{C} / \mathrm{min}$, and then furnace-cooled to room temperature following pressing. This heating schedule is the same as that for the HUP samples. Each HIP sample was given a $1050^{\circ} \mathrm{C}$ thermal treatment for 20 minutes. Samples designated HIP-7 through HIP-18 and A 101-1 through A 101-4 were produced in this manner. One important difference between the HUP and HIP is that the vacuum HUP used a die which was exposed to the vacuum system whereas the HIP samples were sealed in a Ta vessel thus trapping any $P$ vapor that developed during the thermal excursion.

\section{Characterization}

\section{Metallography}

The HIP-5a and HIP-10 compacts were sectioned such that a longitudinal (top face) and transverse (side) portion could be examined by optical metallography. The grain size of HIP-5a was not uniform throughout the compact. In the longitudinal direction HIP-5a has large grains in the 10- used.

\section{Oxygen Determination}

The oxygen content of selected HUP and HIP alloys was determined by neutron activation by Nicolet Imaging Systems-Radiation Processing Group. Values obtained for different alloys are presented in Table 2. These values are consistent with low oxygen n-type Si-Ge alloys prepared by Cook et al. [3].

Table 2. Total oxygen content determined by neutron activation. A92-H is a HUP alloy prepared identically to A92 $\alpha$, the rest are HIP.

\begin{tabular}{cc}
\hline Sample & Oxygen (at.\%) \\
\hline HIP-2 & 0.4 \\
HIP-10 & 0.05 \\
A 101-4 & 0.21 \\
A92-H & 0.2 \\
\hline
\end{tabular}




\section{DISCLAMMER}

Portions of this document may be illegible in electronic image products. Images are produced from the best available original document. 


\section{Chemical Analysis}

Several samples were examined by Inductively Couple Plasma-Atomic Emission Spectroscopy (ICP-AES) and Laser Ionization Mass Spectroscopy (LIMS). ICP-AES was used to determine $\mathrm{P}$ and $\mathrm{Ga}$ contents following hot consolidation. LIMS was used to characterize impurities from sources such as wear debris from the milling vessels.

Table 3 shows the nominal and measured $P$ and $G a$ content in a number of the alloys. It can be seen that the actual amount of $P$ in the HUP alloys varies substantially from the nominal amount. The measured amount of $\mathrm{P}$ is much closer to the nominal amount for the HIP alloys. HIP-7 through HIP-15 show that the final $\mathrm{P}$ content is consistent from one sample to the next. Note that HIP-5b has a lower $P$ content than the remaining HIP samples with the same nominal composition. It is unknown why this sample would have a greater loss of $P$ following hot consolidation.

Table 3. Nominal phosphorous and gallium concentrations. Actual $\mathrm{P}$ and $\mathrm{Ga}$ contents were determined by ICP-AES following hot consolidation.

\begin{tabular}{ccc}
\hline \hline Sample & $\begin{array}{c}\text { Nominal } \\
\text { P at.\% }\end{array}$ & $\begin{array}{c}\text { Actual } \\
\text { P at.\% }\end{array}$ \\
\hline A90 $\alpha$ & 1.25 & 1.1 \\
A91 $\alpha$ & 2.0 & 1.56 \\
A92 $\alpha$ & 1.8 & 1.28 \\
A92 $\beta$ & 1.8 & 1.47 \\
A93 $\alpha$ & 2.5 & 1.87 \\
HIP-2 & 1.8 & 1.56 \\
HIP-5b & 1.5 & 1.13 \\
HIP-7 & 1.5 & 1.36 \\
HIP-10 & 1.5 & 1.39 \\
HIP-12 & 1.5 & 1.36 \\
HIP-15 & 1.5 & 1.36 \\
\hline \hline
\end{tabular}

LIMS data for trace element imprities is presented in Table 4. A101-2 had a slightly lower Fe content than the other A101 compacts, but HIP15 was higher than A101-3 and A 101-4. Portions of the Si-Ge in the A 101-3 compact were in contact with the Ta HIP vessel, but this did not contribute to a higher Ta level in the alloy. The level of Ta contamination is low in all of the alloys. Graphoil facilitates the removal of the sample and does not appear to significantly affect the carbon content.

Table 4. Impurities in MA + HIP alloys as determined by Laser Ionization Mass Spectroscopy. The values are in ppm atomic. Determination of $\mathrm{C}$ is subject to relatively large errors. X-1 refers to a spot near the edge of the compact which was in direct contact with the Ta vessel graphoil. The $x-2$ refers to a spot near the center of the compact.

\begin{tabular}{cccc}
\hline Sample ID & Fe & C & Ta \\
\hline HIP-15 & 420 & 7.3 & 1.9 \\
A101-2 & 170 & 58 & 2.9 \\
A101-3 X-1 & 230 & 25 & 0.34 \\
A101-3 x-2 & 320 & 40 & 0.62 \\
A101-4 & 460 & 10 & 0.10 \\
\hline
\end{tabular}

\section{Properties}

\section{Electrical}

Electrical characterization of the HIP and HUP alloys consisted of room temperature Hall effect measurements, shown in Table 5, and measurements of the temperature dependence of resistivity and Seebeck coefficients to $1000^{\circ} \mathrm{C}$. The high temperature measurements are represented in Table 6 as integrated averages of resistivity, Seebeck coefficient, and power factor in the range of $300^{\circ} \mathrm{C}$ to $1000^{\circ} \mathrm{C}$.

The carrier concentration values given in Table 5 illustrate the change in carrier concentration with sample composition. Nearly optimized figures of merit have been observed in Si-Ge alloys doped with $\mathrm{GaP}$ and $\mathrm{P}$ having a carrier concentration in the $2.5-3.0 \times 10^{20} \mathrm{~cm}^{-3}$ range, as shown, for example, by Scoville et al. [5]. Alloys of the A92 composition fall within or close to that range and this composition was chosen for the first HIP sample, HIP-2. It can be seen in Table 5 that the HIP-2 alloy has a much higher carrier concentration than the corresponding HUP counterparts. The P content of HIP-2 is much higher than that of the HUP alloy A92 as discussed above, which indicates that the HIP approach does not allow the escape of $P$ vapor during consolidation. The nominal phosphorus composition of alloys consolidated by HIPing must therefore be lower than corresponding HUP alloys to compensate for the fact that $P$ is not lost to the vacuum system as it is in the HUP. The HIP-5 alloys had a nominal composition designed to lower the carrier concentration. It can be seen that this was achieved, but as mentioned above, these alloys possessed an anisotropic microstructure. The HIP-7 through HIP-18 compacts were prepared with larger diameter and shorter vessels. It can be seen that the carrier concentration increased for these alloys compared with the HIP- 5 compacts. The HIP- 5 compacts and the HIP-7-HIP-18 compacts have the same composition, but the carrier concentrations are much higher in the 2 " diameter compacts. The behavior of the 2" compacts indicates that the nominal $\mathrm{P}$ level needs to be reduced in order to obtain $\mathrm{Z}$ similar to the laboratory scale samples of $0.94 \times 10^{-3} \mathrm{C}^{-1}$. The carrier concentration of all HIP billets with the Al00 composition is too high, yet the mobility is the highest possible for the given carrier concentration based on extrapolating Dismukes zone leveled Si-Ge data to higher carrier concentration values.

The A101 alloys had a nominal composition intended to lower the carrier concentration to the $2.5 \times 10^{20}$ $\mathrm{cm}^{-3}$ range. Hall measurements of $\mathrm{A} 101-2$ indicated that the target concentration $\mathrm{n}$ of $2.5 \times 10^{20} \mathrm{~cm}^{-3}$ was not attained, but $\mathrm{n}$ was reduced by 14 percent. Although the desired carrier concentration was not achieved, it was lowered to the point where the figure of merit $Z$ was improved, as discussed below. As seen in Table 5, the carrier concentration for the A101 series of alloys decreases with each subsequent sample. The carrier concentration for A101-4 is in the targeted range of $2.5-2.8 \times 10^{20} \mathrm{~cm}^{-3}$, but the mobility is about $10 \%$ lower than the value of 45 expected with this carrier concentration. 
One possible explanation for the low mobility in the A101-3 and A101-4 alloys is that increased wear debris from the grinding media would iron disilicide or germinide scattering centers. The LIMS data in Table 5 tends to supports this idea. Another possability is that the actual HIP temperature was lower than $1130^{\circ} \mathrm{C}$ target. This would lead to smaller grain sizes and reduced mobility due to grain boundary scattering.

Table 5. Room temperature Hall effect properties for HUP and HIP alloys.

\begin{tabular}{cccc}
\hline Sample & $\mathrm{n}\left(\mathrm{x} 10^{2} \mathrm{~cm}^{-3}\right)$ & $\rho(\mathrm{m} \Omega-\mathrm{cm})$ & $\mu\left(\mathrm{cm}^{2} / \mathrm{V}-\mathrm{s}\right)$ \\
\hline A92 $\alpha$ & 2.78 & 0.599 & 37.7 \\
A92 $\beta$ & 3.08 & 0.51 & 40.0 \\
A93 $\alpha$ & 3.10 & 0.50 & 40.4 \\
A95 $\alpha$ & 3.26 & 0.442 & 43.4 \\
A96 $\beta$ & 3.80 & 0.445 & 37.0 \\
A99 $\alpha$ & 3.29 & 0.467 & 40.7 \\
HIP-2, S-1 & 3.53 & 0.46 & 38.8 \\
HIP-2, S-2 & 3.60 & 0.45 & 38.3 \\
HIP-5a & 2.45 & 0.51 & 50.0 \\
HIP-5b & 2.44 & 0.52 & 49.7 \\
HIP-7 & 3.42 & 0.45 & 40.1 \\
HIP-10 & 3.45 & 0.45 & 39.2 \\
HIP-12 & 3.44 & 0.46 & 39.3 \\
HIP-15 & 3.45 & 0.46 & 39.6 \\
HIP-16 & 3.36 & 0.47 & 39.4 \\
HIP-17 & 3.37 & 0.47 & 39.6 \\
HIP-18 & 3.47 & 0.47 & 38.4 \\
A101-2 & 2.95 & 0.51 & 41.4 \\
A101-3 & 2.80 & 0.54 & 41.6 \\
A101-4 & 2.76 & 0.55 & 41.2 \\
\hline \hline
\end{tabular}

The integrated average power factors for the HUP samples from Table 6 range from 31.4 for $\mathrm{A} 92 \alpha$ to 36.6 $\mu \mathrm{W} / \mathrm{cm}-{ }^{\circ} \mathrm{C}^{2}$ for $\mathrm{A} 93 \alpha$. The changes in composition affected the resistivity more than the Seebeck coefficient.

There is also a spread in the power factor data for the HIP alloys. HIP-2 did not duplicate the values in the A92 alloys. As was mentioned above, the carrier concentration of HIP-2 was much higher than that of the HUP counterpart. However, the integrated average power factor is higher for HIP-2 than for A92 $\alpha$. The exact reason for this is not known, but it may be an artifact of the anisotropic microstructure. The loss of $P$ in the hot consolidation of the MA powders plays an important role in properties of the HUP alloys.

Both HIP-5a and HIP-5b have higher integrated average power factors than HIP-2 and A92 $\alpha$. This demonstrates that lowering the carrier concentration to the desired range results in higher performance. Comparing the integrated average resistivity, Seebeck, and power factor of the HIP-5 alloys with the HIP-7 - HIP-17 alloys, it can be seen that both the resistivity and Seebeck increased which lead to an overall decrease in the power factor. Changing the shape of the HIP vessel apparently led to higher $P$ retention which resulted in a higher carrier concentration. These results again demonstrate the neccessity for maintaining the carrier concentration in the correct range.

Table 6. Integrated average electrical high temperature property data from $300-1000^{\circ} \mathrm{C}$ for n-type $\mathrm{Si}_{80} \mathrm{Ge}_{20}$ alloys.

\begin{tabular}{|c|c|c|c|}
\hline Sample & $\begin{array}{c}\langle\rho\rangle \\
(\mathrm{m} \Omega- \\
\mathrm{cm})\end{array}$ & $\begin{array}{c}\langle S> \\
\left(\mu V /{ }^{\circ} \mathrm{C}\right)\end{array}$ & $\begin{array}{c}\langle\mathrm{PF}> \\
\left(\mu W / \mathrm{cm}-{ }^{\circ} \mathrm{C}^{2}\right)\end{array}$ \\
\hline $\mathrm{A} 92 \alpha$ & 1.38 & -205.6 & 31.4 \\
\hline$A 92 \beta$ & 1.17 & -200.5 & 34.7 \\
\hline A93a & 1.17 & -205.5 & 36.6 \\
\hline$A 95 \alpha$ & 1.21 & -203.3 & 35.1 \\
\hline$A 96 \beta$ & 1.15 & -193.9 & 33.4 \\
\hline$A 99 \alpha$ & 1.13 & -195.0 & 34.3 \\
\hline HIP-2 & 1.23 & -204.0 & 34.8 \\
\hline HIP-5a & 1.12 & -215.5 & 41.4 \\
\hline HIP-5b & 1.23 & -217.7 & 38.6 \\
\hline $\mathrm{HIP}-7$ & 1.26 & -200.8 & 32.5 \\
\hline HIP-10 & 1.25 & -201.0 & 32.3 \\
\hline HIP-15 & 1.25 & -200.9 & 33.3 \\
\hline HIP-17 & 1.22 & -201.1 & 33.7 \\
\hline A101-2 & 1.29 & -210.7 & 35.3 \\
\hline A101-3 & 1.32 & -210.3 & 34.4 \\
\hline
\end{tabular}

The A101 series of alloys employed the same 5.08 $\mathrm{cm}$ diameter HIP vessels as HIP-7 through HIP-18, but the P and $\mathrm{Ga}$ levels were decreased in order to lower the carrier concentration. A101-2 demonstrated that the reduction in P from $1.5 \%$ to $1.1 \%$ gave an increase in the average Seebeck coefficient while the resistivity remained relatively unchanged. This led to A101-2 having an average power factor of $35.3 \mu \mathrm{W} / \mathrm{cm}-{ }^{\circ} \mathrm{C}^{2}$ compared to $32-33 \mu \mathrm{W} / \mathrm{cm}-{ }^{\circ} \mathrm{C}^{2}$ for the A100 composition samples. A101-3 had a 5-6\% higher resistivity than A101-2. A portion of this sample was in contact with the Ta HIP vessel during consolidation, however LIMS analysis indicated that an increased Ta content did not affect the electrical properties. LIMS results indicated that this was probably not the case.

\section{Thermal conductivity and Figure of Merit}

Thermal diffusivity was measured using a laser flash diffusivity method as described in Kokos et al. [6]. Table 7 summarizes data for both HIP and HUP samples.

Table 7. Integrated average thermal conductivity $\lambda$ and figure of merit $\mathrm{Z}$ from $300-1000^{\circ} \mathrm{C}$ for HUP and HIP n-type SiGe alloys.

\begin{tabular}{ccc}
\hline \hline Sample & $\langle\lambda\rangle\left(\mathrm{mW} / \mathrm{cm}-{ }^{\circ} \mathrm{C}\right)$ & $\langle\mathrm{Z}\rangle\left(\mathrm{x} 10^{-3}{ }^{\circ} \mathrm{C}^{-1}\right)$ \\
\hline HIP-2 & 44.0 & 0.80 \\
HIP-5a & 44.5 & 0.94 \\
HIP-7 & 38.6 & 0.84 \\
HIP-10 & 39.5 & 0.82 \\
HIP-15 & 38.7 & 0.86 \\
HIP-17 & 39.6 & 0.85 \\
Al01-2 & 38.7 & 0.92 \\
A92 & 37.5 & 0.94 \\
\hline
\end{tabular}

Both HIP-2 and HIP-5a have relatively high thermal conductivities typical of high mobility low oxygen n-type alloys. The thermal conductivities of both of these alloys is nearly identical to that measured in a previous study of HUPed n-type Si-Ge doped with $1.6 \% \mathrm{GaP}$ and a $\mathrm{P} / \mathrm{Ga}$ ratio of $3.125: 1$ with a low oxygen content of 0.2 at. $\%^{3}$. As previously mentioned, the grain structure of HIP-2 and HIP- 
5 a was nonuniform. This may account for the high thermal conductivity values for these two samples. The thermal conductivity for the other HIP alloys using the A100 composition is fairly uniform, ranging from 38.6 to 39.6 $\mathrm{mW} / \mathrm{cm}-{ }^{\circ} \mathrm{C}$.

A101-2 had an integrated average thermal conductivity similar to that of the 2" diameter compacts with the Al00 composition. A101-3 has a higher thermal conductivity than A101-2, as well as a lower power factor as discussed above.

The highest $Z$ attained was on HIP-5a, but that may be due to the anisotropic microstructure. HIP-7 through HIP. 17 had lower integrated average $Z$ 's in the low 0.8 to $0.85 \mathrm{x}$ $10^{-3}{ }^{\circ} \mathrm{C}^{-1}$ range. A $101-2$ had a higher $\mathrm{Z}$, demonstrating the value of lowering the carrier concentration.

\section{Summary}

Two inch diameter compacts of $\mathrm{P}$ and $\mathrm{GaP}$ doped SiGe can be prepared via MA and HIPing. These alloys can attain the high power factors and figures of merit demonstrated by the laboratory scale samples. Due to the differences in hot consolidation between the HIP and HUP, it is impossible to identify one composition that will perform equally under both preparation techniques. Phosphorous loss and solubility is different in both consolidation techniques. Changing the HIP vessel shape can result in changes in carrier concentration.

\section{Acknowledgment}

The Ames Laboratory is operated by Iowa State University for the United States Department of Energy under contract W7405-ENG-82. The advice and assistance of Bernard Beaudry is gratefully acknowledged. Hot isostatic pressing was performed by Lester Reed.

\section{References}

[1]. Sandia National Laboratory Report number SLA-740046, (1974).

[2]. B.A. Cook, B.J. Beaudry, J.L. Harringa, and W.J. Barnett, "The Preparation of SiGe Thermoelectric Alloys by Mechanical Alloying," Proc. of the 24th Intersoc. Energy Conv. Eng. Conf., ed. by W. D. Jackson, Washington, DC: Inst. IEEE, volume 2, 693-700 (1989).

[3]. B.A. Cook, J.L. Harringa, S.H. Han, and B.J. Beaudry, J. Appl. Phys. 72 (1992) 1423-1428.

[4]. B.A.Cook, J.L. Harringa, S.H. Han, and C.B. Vining, J. Appl. Phys. 78 (1995) 5474-5480.

[5]. N. Scoville, C. Bajgar, J.P. Fleurial, and J.W. Vandersande, "Optimization of Heavily Doped SiGe Alloys," Proc. of the XI Int. Conf. on Thermoelectrics, University of Texas at Arlington, Oct 7-9, 1992, 70-73.
[6]. G.B. Kokos, K.A. Gschneidner, B.A. Cook, and B.J. Beaudry, J. Appl. Phys., 66, (1989) 2356. 